\title{
Study of verification and validation of standard welding procedure specifications guidelines for API 5L X-70 grade line pipe welding
}

\author{
Qazi H. A. A.*
}

\author{
Crescent Steel and Allied Products Ltd., Latifabad, Hyderabad, Sindh, Pakistan
}

\author{
Article info: \\ Paper received: \\ The final version of the paper received: \\ Paper accepted online:
}

September 29, 2017

November 25, 2017

November 28, 2017
*Corresponding Author's Address: ahadqazi10@yahoo.com

\begin{abstract}
Verification and validation of welding procedure specifications for X-70 grade line pipe welding was performed as per clause 8.2, Annexure B and D of API 5L, $45^{\text {th }}$ Edition to check weld integrity in its future application conditions. Hot rolled coils were imported from China, de-coiling, strip edge milling, three roller bending to from pipe, inside and outside submerged arc welding of pipe, online ultrasonic testing of weld, HAZ and pipe body, cutting at fixed random length of pipe, visual inspection of pipe, Fluoroscopic inspection of pipe, welding procedure qualification test pieces marking at weld portion of the pipe, tensile testing, guided bend testing, CVN Impact testing were performed. Detailed study was conducted to explore possible explanations and variation in mechanical properties, WPS is examined and qualified as per API $5 \mathrm{~L} 45^{\text {th }}$ Edition.
\end{abstract}

Keywords: welding procedure specification WPS, welding procedure qualification record WPQR, submerged arc welding SAW, american petroleum institute API, american society for testing materials ASTM, tensile test, guided bend test, charpy V-notch impact test.

\section{Introduction}

Welding procedure specification is a standard guideline used to perform a welding action. A WPS is designed and issued by welding engineer and is used by qualified welding operators and welders to perform welding operation so that in each weld, required mechanical properties can be achieved. In a typical WPS, essential variables material grade, voltage (tolerance of less than or equal to $7 \%$ ), current (tolerance of less than or equal to $10 \%$ ), welding speed (tolerance of less than or equal $10 \%$ for automatic welding), heat input (tolerance of less than or equal to $10 \%$ )are given in a range while variables like type of welding process, method of welding electrode diameters, polarity, type of electrode and flux, shield gas type if any used are fixed and cannot be changed once a WPS is qualified. If these are to be changed in any case, a new welding procedure specification is to be made and welding procedure qualification (WPQR) tests have to be performed.

\section{Methodology and results}

To verify mechanical properties mentioned in coil manufacturer mill test certificate, Tensile Test, CVN Impact test samples were cut from as received coil,the testing was performed as per ASTM A370 standard, the testing results are presented in Table 1 which is complying with table 7, clause 9.7, 9.8 and table 8 of API 5L-45 ${ }^{\text {th }}$ Edition. After the verification of mechanical properties, Pipe manufacturing using verified coils was done using these steps, Hot rolled coil was charged using charging lever at Spiral SAW pipe mill where first de-coiling was performed through auxiliary driver rollers, three roller leveling at 140 Bar pressure, five roller leveling at 150 Bar pressure, strip was driven further by main driver rollers at 80 Bar pressure, both edges of plate was milled as per weld geometry design given in WPS, pre-bending of plate, three roller bending at 380 Bar pressure to form pipe, inside and outside welding on pipe was performed by qualified welding operators, Online ultrasonic testing of welds, HAZ and pipe body on full length of pipe to detect defects related to weld, HAZ and pipe body, fluoroscopic examination on full length of pipe was performed to verify soundness of the weld, marking of test pieces on inspected $\mathrm{OK}$ pipe was done, transverse tensile testing on two specimen were performed at $25^{\circ} \mathrm{C}$ to measure ultimate tensile strength picture representation of tensile test specimen, Tensile testing machine, stress-strain diagrams, fracture appearance of tensile test specimen after the test are shown below in Figure 1. Guided bend testing on 4 specimen (cut from weld portion in transverse direction of pipe)two face and two root was performed to bend the specimen at 180 degrees over the mandrel (the mandrel 
dimension was calculated as per clause 10.2.4.6 of API $5 \mathrm{~L}-45^{\text {th }}$ Edition and then to visually inspect for any cracks occur during bending, CVN impact testing on 9 specimen on weld area and 9 specimen on Heat affected Zone area

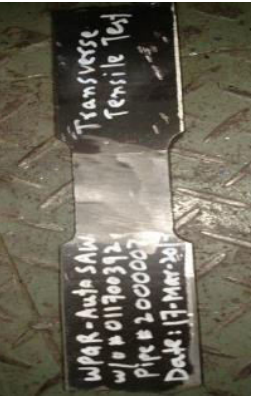

a

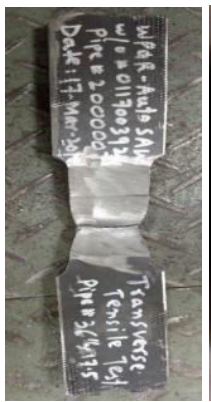

b

at ambient temperature and at $0{ }^{\circ} \mathrm{C}$ was performed to measure energy absorbed in Joules during fracture termed as toughness. Testing results of specimen cut from pipe are presented in Table 2.

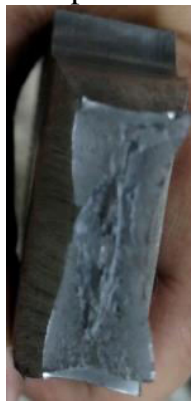

$\mathrm{C}$

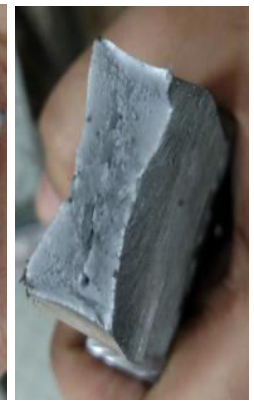

d

Figure 1 - Prepared marked tensile specimen (a); fractured tensile specimen (b); fractured tensile specimen cone appearance (c); fractured tensile specimen cup appearance (d)

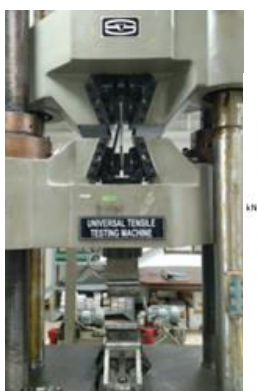

a

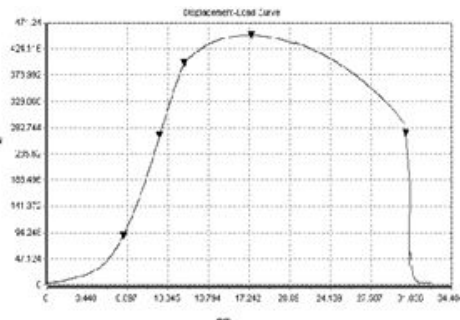

b

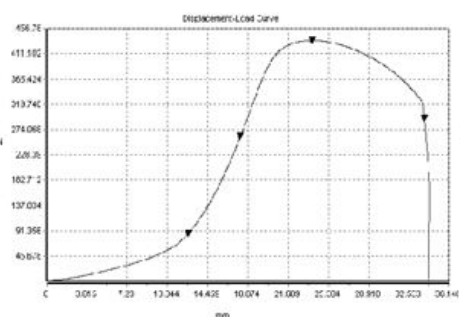

C

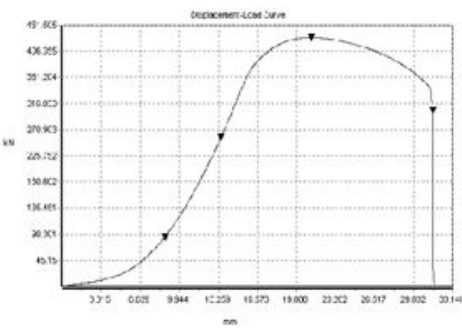

d

Figure 2 - Universal tensile testing machine (a); coil tensile specimen stress-strain diagram (b); automatic machine welding tensile specimen A1 stress-strain diagram (c); automatic machine welding tensile specimen A2 stress-strain diagram (d)

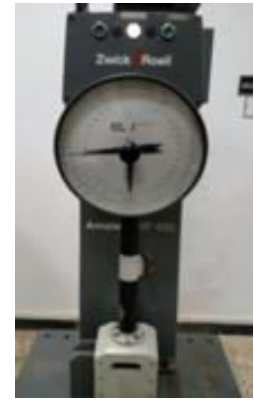

a

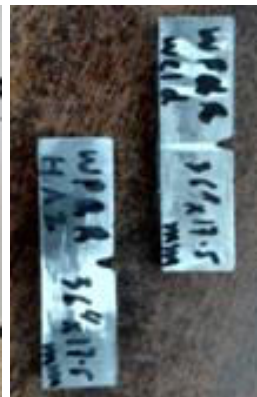

b

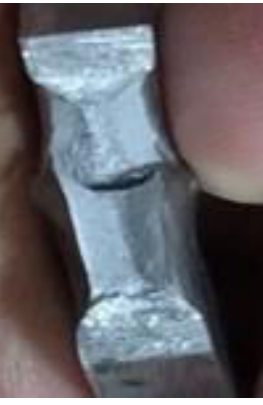

$\mathrm{c}$

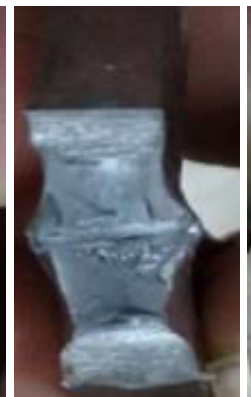

d

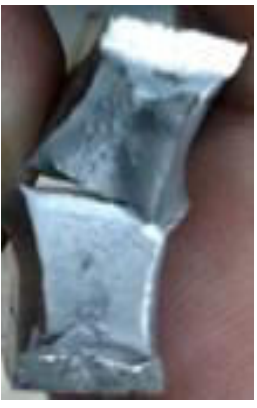

e

Figure 3 - Charpy Impact test machine (a); CVN weld and HAZ test specimen (b); fractured appearance of coil CVN specimen (c); fractured surface of CVN weld specimen (d); fractured surface of HAZ specimen (e)

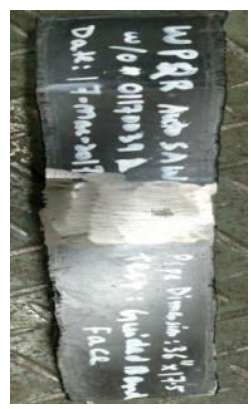

a

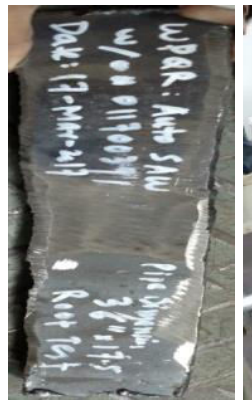

b

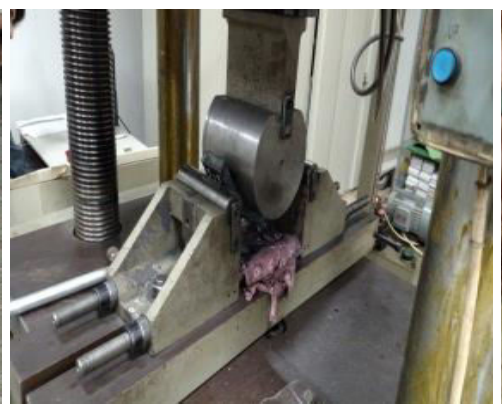

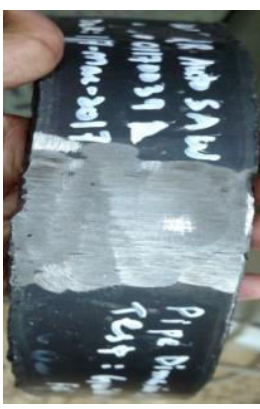

d

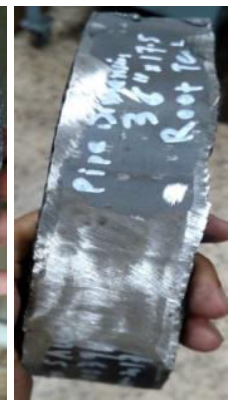

Figure 4 - Prepared guided bend face specimen (a); guided bend root test specimen (b); guided bent test machine (c); face bent specimen showing no crack appearance (d); root bent specimen showing no crack appearance (e) 
Table 1 - Mechanical Properties of As received coil

\begin{tabular}{|c|c|c|c|c|c|c|c|c|c|}
\hline Procedure & Sample & $\begin{array}{c}\text { Sample } \\
\text { Orientation }\end{array}$ & $\begin{array}{c}\text { Test Piece } \\
\text { Average } \\
\text { Thickness } \\
\text { (mm) }\end{array}$ & $\begin{array}{c}\text { Test Piece } \\
\text { Average } \\
\text { Width }(\mathrm{mm})\end{array}$ & $\begin{array}{c}\text { Gauge } \\
\text { Length } \\
(\mathrm{mm})\end{array}$ & $\begin{array}{c}\text { Yied } \\
\text { Strength } \\
(\mathrm{MPa})\end{array}$ & $\begin{array}{l}\text { Ultimate } \\
\text { Tensile } \\
\text { Strength } \\
(\mathrm{MPa})\end{array}$ & $\begin{array}{l}\text { Yield } \\
\text { Ratio } \\
\text { (Yield } \\
\text { Ratio / } \\
\text { UTS) } \\
\end{array}$ & $\begin{array}{c}\text { Elonga- } \\
\text { tion } \\
(\%)\end{array}$ \\
\hline \multirow{2}{*}{$\begin{array}{c}\text { Tensile } \\
\text { Test }\end{array}$} & $\begin{array}{c}\text { Heat } \\
\text { No-Coil } \\
\text { No. } 1\end{array}$ & Transverse & 17.55 & 38.10 & 50 & 590 & 630.82 & 0.90 & $37 \%$ \\
\hline & $\begin{array}{c}\text { Heat } \\
\text { No-Coil } \\
\text { No. } 1 \\
\end{array}$ & Transverse & 17.52 & 38.05 & 50 & 597 & 633.67 & 0.92 & $39 \%$ \\
\hline \multicolumn{10}{|c|}{ Charpy V-Notch Impact Test } \\
\hline \multirow{3}{*}{$\begin{array}{l}\text { Sample } \\
\text { Identifica- } \\
\text { tion }\end{array}$} & $\begin{array}{c}\text { Heat } \\
\text { No-Coil } \\
\text { No. } 1\end{array}$ & \multirow{3}{*}{$\begin{array}{l}\text { Specimen } \\
\text { Size }(\mathrm{mm})\end{array}$} & $10 \times 5 \times 55$ & \multirow{3}{*}{$\begin{array}{c}\text { Energy } \\
\text { Absorbed } \\
\text { Converted } \\
\text { to Full } \\
\text { Sized Spec- } \\
\text { imen } \\
\text { (Joule) }\end{array}$} & 280 & \multirow{3}{*}{$\begin{array}{c}\text { Shear } \\
\text { Fracture, } \\
\%\end{array}$} & \multicolumn{3}{|c|}{$>85$} \\
\hline & $\begin{array}{c}\text { Heat } \\
\text { No-Coil } \\
\text { No. } 2\end{array}$ & & $10 \times 5 \times 55$ & & 270 & & \multicolumn{3}{|c|}{$>85$} \\
\hline & $\begin{array}{c}\text { Heat } \\
\text { No-Coil } \\
\text { No. } 3\end{array}$ & & $10 \times 5 \times 55$ & & 267 & & \multicolumn{3}{|c|}{$>85$} \\
\hline
\end{tabular}

Table 2 - Mechanical Properties of welded test specimens for welding procedure qualification record

\begin{tabular}{|c|c|c|c|c|c|c|c|c|c|}
\hline \multirow[t]{3}{*}{$\begin{array}{c}\text { Tensile } \\
\text { Test }\end{array}$} & $\begin{array}{l}\text { Sample } \\
\text { Identifica- } \\
\text { tion }\end{array}$ & $\begin{array}{l}\text { Sample } \\
\text { Orienta- } \\
\text { tion }\end{array}$ & $\begin{array}{c}\text { Test } \\
\text { Piece } \\
\text { Average } \\
\text { Thick- } \\
\text { ness } \\
(\mathrm{mm}) \\
\end{array}$ & $\begin{array}{l}\text { Test Piece } \\
\text { Average } \\
\text { Width } \\
(\mathrm{mm})\end{array}$ & $\begin{array}{c}\text { Gauge } \\
\text { Lengt } \\
\mathrm{h} \\
(\mathrm{mm})\end{array}$ & $\begin{array}{c}\text { Yied } \\
\text { Strengt } \\
\text { h }(\mathrm{MPa})\end{array}$ & $\begin{array}{c}\text { Ulti- } \\
\text { mate } \\
\text { Tensile } \\
\text { Strength } \\
(\mathrm{MPa})\end{array}$ & $\begin{array}{l}\text { Yield Ratio } \\
\text { (Yield } \\
\text { Ra- } \\
\text { tio/UTS) }\end{array}$ & $\begin{array}{l}\text { Elonga- } \\
\text { tion }(\%)\end{array}$ \\
\hline & Weld 1 & Transverse & 17.58 & 38.10 & 50 & N/A & 651.82 & $\mathrm{~N} / \mathrm{A}$ & $\mathrm{N} / \mathrm{A}$ \\
\hline & Weld 2 & Transverse & 17.52 & 37.99 & 50 & N/A & 651.98 & $\mathrm{~N} / \mathrm{A}$ & $\mathrm{N} / \mathrm{A}$ \\
\hline \multicolumn{10}{|c|}{ Charpy V- Notch Impact Test } \\
\hline \multirow{6}{*}{$\begin{array}{l}\text { Sample } \\
\text { Identifica- } \\
\text { tion }\end{array}$} & Weld 1 & \multirow{6}{*}{$\begin{array}{l}\text { Specimen } \\
\text { Size- Half } \\
\text { Size } \\
\text { Reduced } \\
\text { Specimen } \\
(\mathrm{mm})\end{array}$} & $10 \times 5 \times 55$ & Energy & 212 & \multirow{6}{*}{$\begin{array}{c}\text { Shear } \\
\text { Fracture, } \\
\%\end{array}$} & \multicolumn{3}{|c|}{$>85$} \\
\hline & Weld 2 & & $10 \times 5 \times 55$ & Absorbed & 240 & & \multicolumn{3}{|c|}{$>85$} \\
\hline & Weld 3 & & $10 \times 5 \times 55$ & Convert- & 224 & & \multicolumn{3}{|c|}{$>85$} \\
\hline & HAZ 1 & & $10 \times 5 \times 55$ & ed & 268 & & \multicolumn{3}{|c|}{$>85$} \\
\hline & HAZ 2 & & $10 \times 5 \times 55$ & to Full & 252 & & \multicolumn{3}{|c|}{$>85$} \\
\hline & HAZ 3 & & $10 \times 5 \times 55$ & $\begin{array}{c}\text { Sized } \\
\text { Specimen } \\
\text { (Joule) }\end{array}$ & 264 & & \multicolumn{3}{|c|}{$>85$} \\
\hline \multicolumn{10}{|c|}{ Guided Bend Test } \\
\hline \multirow{2}{*}{$\begin{array}{l}\text { Sample } \\
\text { Identifica- } \\
\text { tion }\end{array}$} & Face Bend & \multirow{2}{*}{$\begin{array}{l}\text { Specimen } \\
\text { Size }\end{array}$} & $\begin{array}{c}300 \times 50 \\
\mathrm{~mm}\end{array}$ & \multirow{2}{*}{\multicolumn{2}{|c|}{$\begin{array}{l}\text { Visual Condition } \\
\text { of specimen } \\
\text { after bend test }\end{array}$}} & \multicolumn{4}{|c|}{$\begin{array}{l}\text { No cracks and open to surfaces } \\
\text { defects observed }\end{array}$} \\
\hline & Root Bend & & $\begin{array}{c}300 \times 50 \\
\mathrm{~mm}\end{array}$ & & & \multicolumn{4}{|c|}{$\begin{array}{l}\text { No cracks and open to surfaces defects } \\
\text { observed }\end{array}$} \\
\hline
\end{tabular}

From the Table 1, it has been observed that tensile strength value of as received plate was lower as compared to the results obtained from test weld test pieces. In addition to the filling material added during welding, since welding is a thermal fusion process, material which was joined through welding had to gone though heating and cooling cycles that caused the mechanical properties of the material altered as in our case from Table 2 , it can be seen tensile strength of the welded specimen has been increased. 


\section{Conclusion}

The weld metal is comparatively stronger, and the joint properties are controlled by weld metal chemical composition and microstructure. Although the welding wire is generally of a composition that matches that of the parent metal yet alloying elements are intentionally added in welding wires to improve final weldment mechanical properties major improvement in mechanical properties are observed due to the heating and cooling cycle that a material encountered during welding process thus grain structure of the material has been refined which in result increases ultimate tensile strength and impact toughness.

Mechanical Testing has been done against the welding joint made as per welding procedure specification, the results are complying with American Petroleum Institute requirement for weld joint of X-70 grade line pipe PSL 2 welding procedure specification has been validated by Quality Control department and permission to proceed has been given to the production department to continue welding practice as per approved welding procedure specification.

\section{References}

1. API 5L $45^{\text {th }}$ Edition Specification for Line Pipe. American Petroleum Institute, 2013.

2. ASTM A370. Standard Test Methods and Definitions for Mechanical Testing of Steel Products. ASTM International.

3. ASME Section IX. QW Welding. ASME International, 2017.

4. Rampaul, H. (2003). Pipe Welding Procedures. Industrial Press, New York, USA.

5. Coryell, K. W. (2005). The Professional's Advisor on WPS/PQR Cross Reference Tables. American Welding Society, Miami, USA.

6. Pullarcot, S. K. (2002). Practical Guide to Pressure Vessel Manufacturing. CRC Press, Boca Raton, USA.

7. Jeffus, L., \& Baker, B. (2016). Pipe Welding. Delmar-Cengage Learning, Inc., Montgomery, USA.

8. Kikani. P., \& Shah S. (2015). Comparison of WPS and PQR for Pressure Vessel Welding. LAP Lambert Academic Publishing, Saarbrucken, Germany. 